\title{
Liquid Cell TEM of Al Thin Film Corrosion under Potentiostatic Polarization
}

See Wee Chee ${ }^{1,2}$, Jeung-Hun Park ${ }^{3}$, Ainsley Pinkowitz ${ }^{1}$, Brent Engler ${ }^{1}$, Frances M. Ross ${ }^{4}$, David Duquette $^{1}$ and Robert Hull ${ }^{1}$

1. Department of Materials Science and Engineering, Rensselaer Polytechnic Institute, Troy, NY 12180, USA.

2. Center of BioImaging Sciences, Department of Biological Sciences, National University of Singapore, Singapore 117557.

3. Department of Materials Science and Engineering, University of California Los Angeles, Los Angeles, CA 90095, USA.

${ }^{4}$ IBM TJ Watson Research Center, Yorktown Heights, NY 10598, USA

Controlling the detrimental effects of metal corrosion is a key concern in many aspects of our modern society. In research looking to elucidate the mechanisms that govern corrosion behavior of a metal, electrochemical techniques play an essential role [1]. While it has been shown that liquid cell transmission electron microscopy can visualize in situ the corrosion structures that form in metal thin films immersed in aqueous media [2], integration with electrochemical measurements is needed if crucial information is to be obtained about the underlying processes. Electrochemical cells for in situ TEM have seen significant development due to the needs of battery research [3], but there are technical challenges in translating the method to corrosion research. In particular, the working electrode in most corrosion experiments is the metal of interest itself. However, in the electrochemical cells for liquid cell TEM, the sample is attached to pre-patterned thin film electrodes, commonly made of Au or Pt. The exposed contact between an active metal and a noble metal can lead to rapid galvanic corrosion of the active metal (see Figure 1).

Here, we will describe experiments in which a blanket Al thin film (100 nm thick) is deposited over one of the two blank chips (without pre-patterned electrodes, see Figure 2(a)) that make up the microfluidic cell in a Hummingbird Scientific liquid flow holder. The film makes contact with one of the three metal leads as the working electrode and the other two leads are used as reference and counter electrodes respectively. The electrolyte is a mixture of $0.1 \mathrm{M} \mathrm{Na}_{2} \mathrm{SO}_{4}$ and $0.001 \mathrm{M} \mathrm{NaCl}$ dissolved in de-ionized water. The solutions are de-aerated by bubbling nitrogen gas overnight and fluid flow is maintained at 5 $\mu 1$ per min using a syringe pump. Potentiostatic polarization is carried out with a Gamry Reference 600 potentiostat with concurrent observations recorded at TV rate (30 frames per sec) in a FEI CM30 TEM operated at $300 \mathrm{kV}$.

Figure 2 (c)-(e) shows successive images in which the dissolution of individual grains (seen with dark diffraction contrast) in the film is observed as the potential is raised from $-200 \mathrm{mV}$ in $0.5 \mathrm{mV} / \mathrm{s}$ steps. As expected, the dissolution takes place in the anodic portion of the curve. These experiments demonstrate direct observation of localized corrosion under potentiostatic control, but the design suffers from two issues. First, corrosion is most rapid at the contact between the film and metal lead, which can lead to loss of electrical contact during an experiment. Second, corrosion takes non-uniformly over the entire film, making it challenging to quantitatively correlate the electrochemical information with the observed phenomena at the silicon nitride window. Strategies to mitigate these issues will be discussed. 


\section{References:}

[1] G.S. Frankel and M. Rohwerder in "Encyclopedia of Electrochemistry, Volume 4, Corrosion and Oxide Films" ed. M. Stratmann and G.S. Frankel (Wiley-VCH, Weinheim), p. 687.

[2] S.W. Chee et al. Microsc. Microanal. 20 (2014) p. 462, Chem. Comm. 51 (2015), p. 168

[3] C.M. Wang, J. Mater. Res. 30 (2015), p.326.

[4] The authors acknowledge funding from the National Science Foundation, grant number DMR1309509.

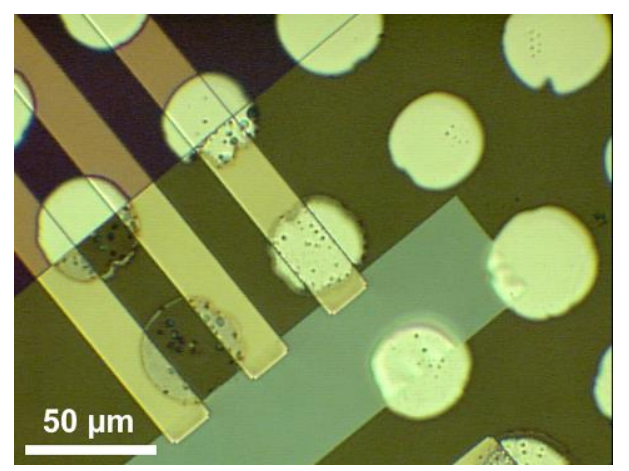

Figure 1. Light microscope image of $\mathrm{Al}$ film deposited through a shadow mask over Au electrodes of an electrochemical chip after 12 mins in $0.1 \mathrm{M} \mathrm{NaCl}$ solution. Notice that areas in contact with bare $\mathrm{Au}$ experienced significant $\mathrm{Al}$ dissolution.

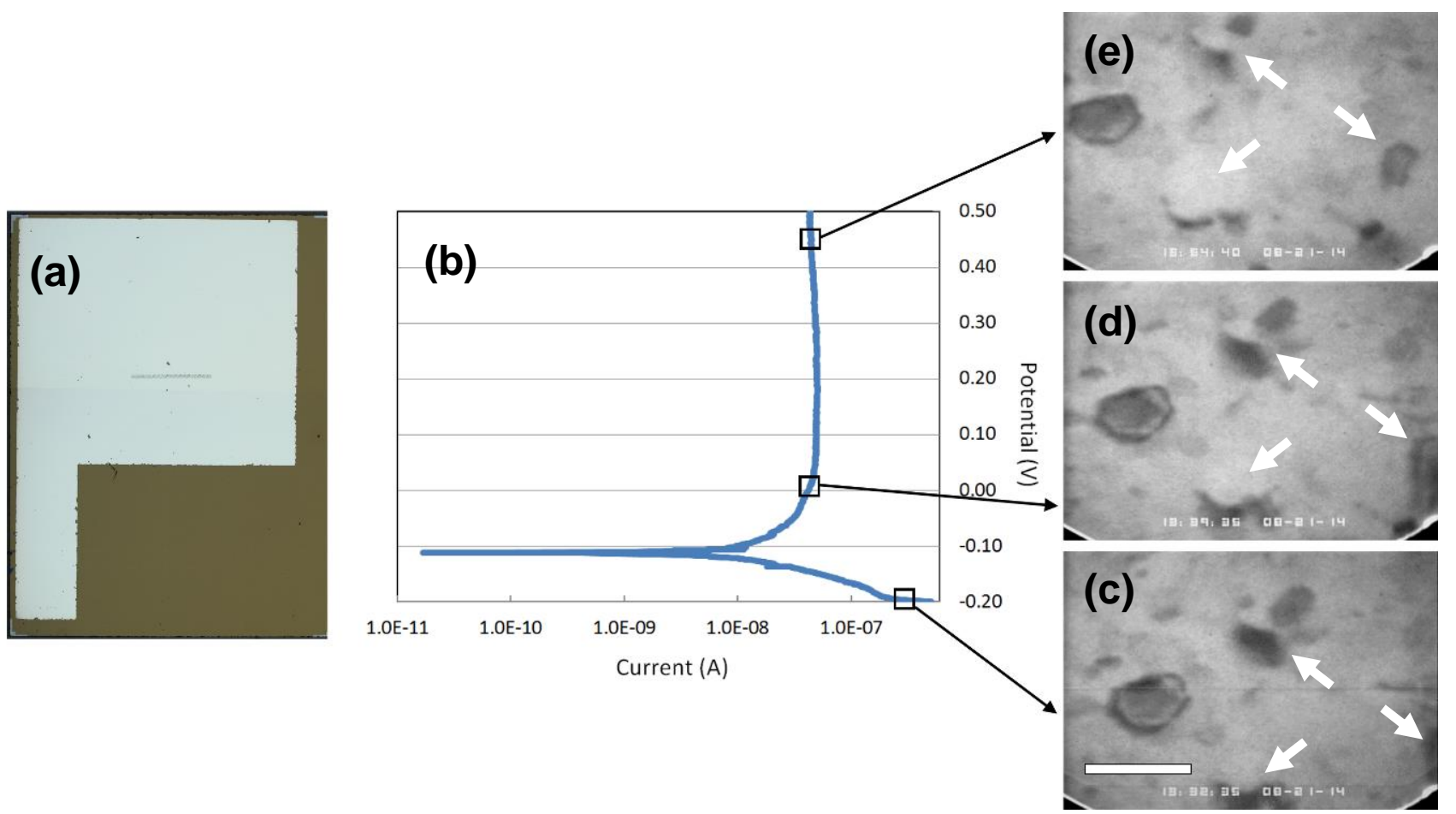

Figure 2. (a) Light microscope image of the Al working electrode (b) Polarization curve of an Al thin film in $0.1 \mathrm{M} \mathrm{Na}_{2} \mathrm{SO}_{4}$ and $0.001 \mathrm{M} \mathrm{NaCl}$ solution. (c-e) Snapshots of film microstructure at different points of the polarization (from $-200 \mathrm{mV}$ to $500 \mathrm{mV}$ ) showing the dissolution of single grains (indicated with white arrows). Note that this is the second time that the sample has been polarized in the solution. Scale bar is $50 \mathrm{~nm}$. 